\title{
ANAESTHESIA FOR UNUSUAL PRESENTATION OF RHINOSPORIDIOSIS: A CASE REPORT
}

\author{
Gurudas Kini', Gopalakrishna ${ }^{2}$, Daniel Thomas ${ }^{3}$ \\ Professor of Anesthesiology, Kasturba Medical College, Manipal, India ${ }^{1}$, Assistant \\ Professor, Department of Anesthesiology, Kasturba Medical College, Manipal, India ${ }^{2}$ \\ Assistant Professor, Department of Anesthesiology, Kasturba Medical College, \\ Manipal, India ${ }^{3}$ \\ * Corresponding author: gopalnayak2004@yahoo.co.in
}

Key words: Bronchial rhinosporidiosis: Excision, laser, cautery, one lung ventilation

Rhinosporidiosis is a rare chronic granulomatous infection caused by Rhinosporidiosis seeberi. Rhinosporidiosis is endemic in parts of Indian subcontinent, Sri Lanka and Bangladesh. They produce large polypoidal lesion that are friable, bleed profusely during resection and have a high tendency to recur. The common site of occurrence is nose and nasopharynx but can present at unusual sites. The occurrence at tracheobronchial tree is rare but can pose challenging problems for surgical excision as well as anaesthetic management.

We are presenting a case of a 30 year old man who had (delete a) recurrent nasopharyngeal Rhinosporidiosis with progressive dyspnoea who was found to have right bronchial involvement. The lesion was excised successfully under general anesthesia using KTP 532 laser and bipolar cautery via tracheostoma. Patient had another surgery for nasopharyngeal lesion after 2 weeks. There was no recurrence of the lesion noted after two year follow up.

Summary: We report a case of right bronchial rhinosporidiosis which was excised using laser/ electric cautery successfully under general anaesthesia using one lung ventilation without any complications and no recurrence on two years follow up. 


\section{Case report:}

A 30 year old male from southern India presented with 2 months history of progressive dyspnoea, haemoptysis and

nose block. He had recurrent nasopharyngeal rhinosporidiosis since childhood for which eight surgical procedures were performed and on a few occasions even (delete he) required blood transfusions. Patient had childhood portal hypertension with oesophageal varices for which he received sclerotherapy. He was an exsmoker but not an alcoholic.

On examination, patient was quite alert, well oriented and not tachypnoeic. There were multiple red granular masses involving bilateral nasal cavities and oropharynx behind the soft palate. There was a decreased breath sound on right side with occasional rhonchi. CT scan of the thorax showed a mass occluding the right main and intermediate bronchi with the involvement of adjacent carina with early features of collapse of right lung. Ultrasound examination of abdomen showed features of cirrhosis of liver with gross splenomegaly. The blood investigations were within normal limits. This patient was taken up for the laser / electric cautery assisted resection of the bronchial rhinosporidiosis under general anesthesia. The standard nil per oral guideline was followed. Patient had received anti-aspiration prophylaxis with T. Pantaprazole $40 \mathrm{mg}$ and $\mathrm{T}$. Metoclopramide $10 \mathrm{mg}$ (a) night before and on the day of the procedure. The preoperative salbutamol nebulisation and T. deriphylline was continued.

After shifting to the operating room all basic standard monitors were connected and his baseline vitals were: heart rate76 minute $^{-1}$, arterial pressure (non invasive) - 120/76 mmHg, $\mathrm{SpO}_{2}-95 \%$ on room air. Two 18 Gauge intra venous access were secured in left upper limb. Right radial artery was cannulated and kept hep-locked. The upper airway was topically anaesthetised with local anaesthetic in semi-recumbent position. An awake fibreoptic bronchoscopy was done which showed a movable mass obstructing the right bronchus without tracheal involvement. Following bronchoscopy, the patient developed severe dyspnea with absence of breath sounds on right side of chest and $\mathrm{SpO} 2$ (was) dropped to $88 \%$ on room air. Patient was kept in right lateral and head up position followed by salbutamol nebulisation. Patient was symptomatically better after 30 minutes. Then, he was preoxygenated with $100 \%$ oxygen for 3 minutes and anaesthesia was induced with sevoflurane in oxygen and $50 \mu \mathrm{g}$ of intravenous fentanyl. As the patient tolerated inhalational induction, anaesthesia was deepened with isoflurane and intravenous propofol boluses. Patient was intubated orally with $7.0 \mathrm{~mm}$ internal diameter (ID) cuffed tube without any relaxant and grade I laryngoscopy. Anaesthesia was maintained with isoflurane in oxygen while doing the tracheostoma. A sterile 6.0mmIDMLS (Micro laryngeal surgery) tube was passed into left bronchus through the tracheostoma by surgeons, position was confirmed by us and oral endotracheal tube was removed. Surgeons could not get enough space to operate through the tracheostoma, so oral reintubation was done with 6.0 mmID MLS tube which was passed into the left bronchus under fibreoptic guidance under anaesthesia. Subsequently, patient was paralysed with vecuronium bromide. Through out the surgical resection, one lung ventilation was used and anaesthesia was maintained with oxygen, isoflurane, vecuronium, fentanyl and intravenous infusion of propofol. There was no usage of nitrous oxide or air through out the procedure and no desaturation under anaesthesia. The resection of the growth was done using KTP 532 laser and 
bipolar cautery through the tracheostoma. The procedure was uneventful with minimal bleeding. At the end of the surgical procedure, residual neuromuscular blockade was reversed and extubated. A cuffed tracheostomy tube was inserted. Oxygen supplementation using T-piece was given. Patient was very comfortable in post operative period.

\section{Discussion:}

Rhinosporidiosis caused by the aquatic parasite Rhinosporidium seeberi, is a common infection in the tropics, with the highest prevalence in southern India and Sri Lanka ${ }^{1}$. The condition commonly involves the nose, nasopharynx, lacrimal sac, larynx and conjuctiva in that order of frequency ${ }^{2}$. The infection is transmitted to humans by direct contact with spores via dust, infected clothing or swimming in stagnant water contaminated with spores 2 . The involvement of tracheobronchial tree is very rare, having been reported in occasional case reports ${ }^{2,3,4,5,6,7,8}$. A few cases of laryngeal rhinosporidiosis were reported in literatures but not the bronchial involvement. These two sentences seem mutually contradictory. Here, we present a case of recurrent nasopharyngeal rhinosporidiosis with right bronchial involvement which was successfully excised under general anesthesia (successfully). The bronchial involvement could be more likely due to secondary implantation following the previous surgery. It is usually agreed that surgical excision is the treatment of choice for rhinosporidial mass and external surgery should be avoided for the fear of local implantation.

The preoperative CT scan of chest of our patient showed mass in the right main bronchus partly involving to the adjacent carina. In view of this, awake fibreoptic bronchoscopy under topical anesthesia was done to assess the extent of involvement before anaesthetising the patient. It showed movable mass arising from right bronchus popping up into the adjacent carina. Following fibreoptic bronchoscopy, patient had dyspnoea with absence of breath sounds on right of chest probably due to the movable mass partly occluding the carina in the supine position as shown by the preoperative CT-scan. Then he was kept in right lateral with head up position and with salbutamol nebulisation and he was symptomatically became better in 30 minutes, GRAMMAR! obviously due to the mass moving away from the carina and lumen of the right main bronchus. To prevent the dislodgement of the mass distally during positive pressure ventilation, we avoided usage of relaxant till we could secure the endotracheal tube into left main stem bronchus. In this case, we guided $6.0 \mathrm{mmID}$ MLS tube into the left main bronchus using fibreoptic bronchoscope to avoid spillage / contamination of other lung and one lung ventilation was used through out the procedure. The passage of MLS tube through the oral route into the left main bronchus provided more room for the surgeon to gain access to the mass through the tracheostoma. The surgery lasted for around 2 hours. The surgical excision was done through tracheostoma route to avoid the external approach for the fear of implantation. The CTVS surgeon was kept as a standby in case bleeding from the lesion cannot be controlled externally. After excision of the mass, patient showed a dramatic improvement with regard to his dyspnoea which was obvious during the recovery period. Two weeks later, patient was taken up for nasopharyngeal lesion excision under general anaesthesia which was uneventful. There was no recurrence noted on two year follow up of this patient.

Conclusion: The bronchial presentation of Rhinosporidiosis may be secondary to 
implantation from the previous surgeries for nasopharyngeal rhinosporidiosis. In our case, patient had bronchial Rhinosporidiosis after repeated surgery for nasopharyngeal Rhinosporidiosis. We feel that adequate precautions during the previous surgeries could have prevented this unusual presentation of Rhinosporidiosis.

\section{References:}

1. Fredricks DN, Jolly JA, Lepp PW, Kosek,

$J C$, Relman DA. Rhinosporidum seeberi: a human pathogen from novel group of aquatic protistan parasites: Emerg Infect Dis 2000; 6: 273-82.

2. Reka P, Thomas B, Joseph $M$ et al. Tracheal rhinosporidiosis. Thorac Cardiovasc Surg 2006;132:718-719
3. Thomas T, Gopinath N, Betts RH. Rhinosporidiosis of the Bronchus. $\mathrm{Br} \mathrm{J}$ Surg 1956; 44:316-19.

4. Shah AK, Ingle MV. Tracheal rhinosporidiosis. Journal of Post Graduate Medicine 1985; 31(4) 210-211

5. Grewal GS, Rangam CM. Rhinosporidiosis of the trachea: an unusual case. J Laryngol Otol 1959; 73: 849-52.

6. Pillai OS. Rhinosporidiosis of the larynx. J laryngol Otol 1974; 88:77-80.

7. Banerjee SB, Sarkar A, Mukharjee $S$, Bhownik A. Laryngeal rhinosporidiosis. J Indian Med Assoc 1996; 94:148,150.

8. Kumar S, Mathew J, Cherian V, Rozario $R$. Kurien $M$. laryngeal rhinosporidiosis: Report of a rare case. ENT Journal. 2004; 63(8): 568-570.
Workshop

on

\section{Primary Trauma Care}

Venue -

Trauma Lecture Hall

National Hospital, Sri Lanka

Date

on Thursdays 